\title{
The physical properties of the compact star forming region N88A and its neighbourhood in the SMC ${ }^{\star}$
}

\section{Excitation and abundances}

\author{
G. Testor ${ }^{1, \star \star}$, J. L. Lemaire ${ }^{2, \star \star \star}$, and D. Field ${ }^{3, \star \star \star}$ \\ 1 LUTH, UMR 8102 du CNRS, Observatoire de Paris, 92195 Meudon, France \\ e-mail: gerard.testor@obspm.fr \\ 2 LERMA, UMR 8112 du CNRS, Observatoire de Paris, 92195 Meudon, France and Université de Cergy-Pontoise, \\ 95031 Cergy Cedex, France \\ e-mail: jean-louis.lemaire@obspm.fr \\ 3 Department of Physics and Astronomy, Århus University, 8000 Århus C, Denmark \\ e-mail: dfield@phys.au.dk
}

Received 1 April 2003 / Accepted 12 June 2003

\begin{abstract}
We present optical, near-infrared (NIR) images and spectra of the compact, dusty and complex HII region N88A in the SMC. The data were obtained mainly in the visible with the Boller \& Chivens (B\&C) spectrograph at the ESO $1.5 \mathrm{~m}$ telescope and recently with the infrared spectrometer and array camera (ISAAC) at the VLT, around $2 \mu \mathrm{m}$. The NIR observations identified a region emitting in $\mathrm{H}_{2}$, both in and around the two major components of N88. Using long-slit spectra obtained in the 3600-10 $100 \AA$ A wavelength range we have re-determined the key physical parameters of electron temperature and densities. The spectral type of the complex exciting source of N88A has been classified as an O6-O8 V, using He and $\mathrm{H}$ lines in the visible and NIR, with the HeI $1.7 \mu \mathrm{m} / 2.11 \mu \mathrm{m}$ line ratio in the $K$ band spectrum, suggesting that N88A is effectively ionized by a source of type $<\mathrm{O} 7 \mathrm{~V}$. However, from reddening correction and the temperature determined by photoionization model calculations, the ionizing source should also contain a very early type $\mathrm{O}$ star. A comparison with a recent photometric study shows that the $V$ magnitude of the exciting source of N88A has previously been overestimated. The chemical abundances of He, $\mathrm{O}, \mathrm{N}, \mathrm{Ne}, \mathrm{S}$, and Ar, from emission-line intensities, are also derived. Abundancies are consistent with the average abundances for SMC HII regions.
\end{abstract}

Key words. galaxies: Magellanic Clouds - ISM: individual objects: N88A (SMC) - ISM: HII regions - ISM: abundances infrared: ISM - stars: early-type

\section{Introduction}

The recent discovery of the heavily reddened compact HII region N26A-B (Testor 2001) in the SMC has strengthened interest in continuing a detailed investigation of N88A, a similar neighbour region extensively studied since its discovery by Testor \& Pakull (1985). Like N26A-B, N88A has a close component B, only $5^{\prime \prime}$ distant and less excited. N88A is classified as a member of a rare class of compact HII regions $(F W H M \leq 2 \mathrm{pc}$ in $\mathrm{H} \beta$ ) (Testor 2002), generally associated with more extended HII regions detected by Henize (1956).

Send offprint requests to: G. Testor, e-mail: testor@obspm.fr and L. Lemaire, e-mail: lemaire@obspm. fr

* Based on observations obtained at the European Southern Observatories, La Silla and El Paranal, Chile.

$\star \star$ Visiting astronomer at la Silla.

$\star \star \star$ Visiting astronomer at Paranal.
N88A is strongly excited and is among the brightest objects in the Magellanic Clouds (MCs). The first highly excited compact HII region in the MCs, now generically named HEBs, was discovered two decades ago (Heydari \& Testor 1982) in a study of individual regions in a low metallicity environment. In spite of their small angular size, as the clouds are sufficiently close, HEBs are the best candidates for the study of individual star forming regions, both in detail and globally. HEBs should be the final stage of ultra compact HII regions where new massive stars are forming (Churchwell 1990). Up to now, eight HEBs have been found in the MCs. Save for N26 A-B, their characteristics are reviewed by Heydari et al. (2002). Israel \& Koorneef $(1988,1991)$ have shown that the N88 region has a strong IR continuum with additional emission from shocked $\mathrm{H}_{2}$. Wilcots (1994a,b) has presented radio continuum imagery, optical imagery and spectroscopy as well as $U B V$ photometry. N88A was intensively studied by Kurt et al. (1999) using 
UV, optical and HST observations both in imaging and spectroscopy. Kurt et al. described this region, formed from a dusty molecular cloud, as a very young HII region, cut by a dark band and excited by at least two stars. They noted that the reddening, especially in the UV, must be different from the SMC extinction and presented an accurate determination of elemental abundances. These were found to be similar to values in other recent studies, except that $\mathrm{C}$ was found overabundant. Subsequently, thanks to the refurbished HST, Heydari et al. (1999, hereafter HM99) give a detailed description of the complex morphology of N88A, especially of the bright and dusty central region where the extinction is estimated to have a peak $A_{V}$ of $\sim 3.5$ mag at the position of the dark lane. In a region of extent $\sim 0.3^{\prime \prime}$, west of the dark lane, HM99 found three faint exciting stars arriving on the zero age main sequence that could belong to the so called $V z$ luminosity class (Walborn \& Parker 1992). However, despite such detailed studies, little can be said about the massive stars in N88A except on the basis of the $\mathrm{H} \beta$ flux. The faint main exciting star remains unidentified. Up to now, no spectrum of the ionizing cluster has been published. Vermeij \& van der Hulst (2002) have revisited the physical structure of the region. They suggest that abundance determinations suffer from a number of uncertainties in line fluxes mainly due to the extinction correction, a point which we confirm here. Massey (2002) presents an accurate $U B V$ photometry of the region, except for the A and B components of N88.

It is evident from these numerous studies of N88 that many fundamental quantities await accurate determination. In the present paper we work towards this goal, presenting results, accumulated over several years, involving optical and NIR spectroscopic and imaging data, largely for the bright region N88A. The paper is organized as follows: Sect. 2 gives a brief description of old and new data as well as the photometry of N88A and its nearby environment. Section 3 briefly outlines the morphology of the region and Sect. 4 the extinction and the spectral classification of the ionizing source of N88A, obtained from Lyman photons and from spectroscopy. In addition physical parameters such as electron density, temperature and mass of ionized gaz are given. Section 5 describes the chemical abundances followed by a summary of results in Sect. 6 .

\section{Observations and data reduction}

\subsection{Spectroscopy}

Table 1 lists all the observations of long-slit optical and NIR spectra obtained during four observing runs at the ESO $1.5 \mathrm{~m}$ telescope with the B\&C spectrograph, as follows:

- In December 1988 a dispersion of $114 \AA \mathrm{mm}^{-1}$, giving a spectral coverage from 3400 to $5400 \AA$ and a $F W H M$ of $4.8 \AA$.

- In October 1991 a high resolution $2000 \times 2000$ CCD Ford at $15 \mu \times 15 \mu$ per pixel corresponding to $0.7 \times 0.7$ on the sky and a grating with a dispersion of $114 \AA \mathrm{mm}^{-1}$, giving a large spectral coverage from 3600 to $7400 \AA$ and a $F W H M$ of $4.8 \AA$.

- In January and December 1995 the same $2000 \times 2000$ CCD Ford but with a holographic grating, giving a reciprocal dispersion of $30 \AA \mathrm{mm}^{-1}$. In the range $\lambda \lambda 4000-5200 \AA$, this grating yielded a spectral resolution $\sim 1.1 \AA$.
Table 1. Journal of the spectrographic observations conducted with the $\mathrm{B} \& \mathrm{C}$ spectrograph.

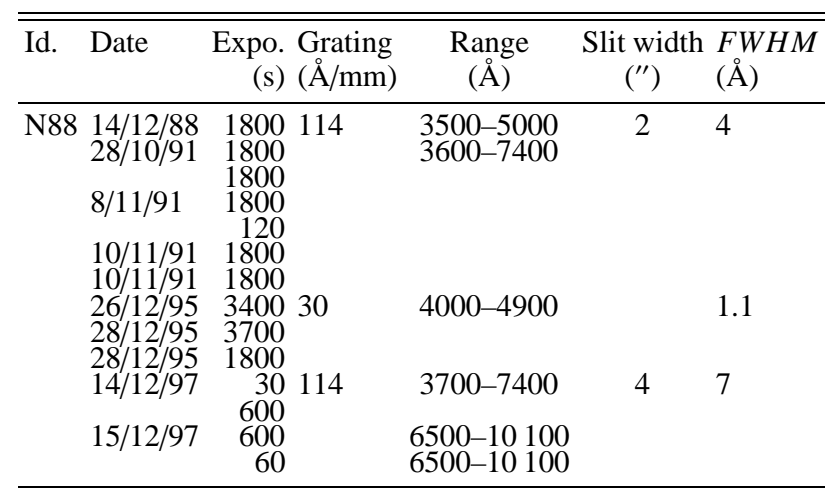

- In December 1997 the Loral $2000 \times 2000 \times 15 \mu$ m chip with two different gratings of dispersion $114 \AA \mathrm{mm}^{-1}$ from 3600 to $7400 \AA$ and of $116 \AA \mathrm{mm}^{-1}$ from 6500 to $10100 \AA$ giving a $F W H M$ of $\sim 7.3 \AA$. Both short and long exposures were taken because of the problem of overexposure in the very strong [O III] $\lambda 5007$ and $\mathrm{H} \alpha$ lines. The $2^{\prime \prime}$ and $4^{\prime \prime}$ long-slit widths were oriented $\mathrm{E}-\mathrm{W}$.

Spectroscopic data were reduced mainly with the Midas software package LONG available at Paris-Meudon Observatory. Corrections such as flat-fielding and sky substraction were applied in the standard manner. The spectra were wavelength calibrated with a helium-argon arc. The flux was calibrated using observations of the spectrophotometric standard stars Feige 24 and 110 (Oke 1974).

\subsection{Imaging of $N 88 A$}

The Infrared Spectrometer and Array Camera (ISAAC) installed on the ESO-VLT UT1 has been used for imaging in the infrared. Details of the data and reduction methods will be described in a subsequent (Paper II). Images of N88A were obtained using the $K$ s broad band filter (Fig. 1a) and two narrowband filters at $2.13 \mu$ and at $2.19 \mu$, both with $1.3 \%$ bandwidth, where data for the former filter are shown in Fig. 1b. For the bright objects, the background continuum is dominant at both wavelengths. Below, we refer to these bright images in the continuum as cont $2.13 \mu$ and cont $2.19 \mu$ respectively. In addition, in the ionized region a Helium line may appear at $2.11 \mu \mathrm{m}$, in the blue wing of the $2.13 \mu \mathrm{m}$ filter. On the faint objects in the $2.13 \mu \mathrm{m}$ filter, the $1-0 \mathrm{~S}(1) \mathrm{H}_{2}$ line at $2.121 \mu \mathrm{m}$ may in some cases be seen in addition to the continuum (see Sect. 3.1). The $2.19 \mu \mathrm{m}$ filter, which does not contain any $\mathrm{H}_{2}$ lines, shows only the continuum emission.

In the visible, a continuum image (Fig. 1c), taken through a narrow-band filter $(\delta \lambda=100 \AA)$ centered at $5300 \AA\left(\operatorname{cont}_{\lambda 5300}\right)$, was also used to compare the optical and NIR continuum. This cont $_{\lambda 5300}$ image was obtained from a CCD $(500 \times 300$ chip, pixel size $=0.47)$, attached to the Danish $1.5 \mathrm{~m}$ telescope at la Silla. Table 2 summarizes the $\log$ of CCD frames. The images were reduced using MIDAS. 


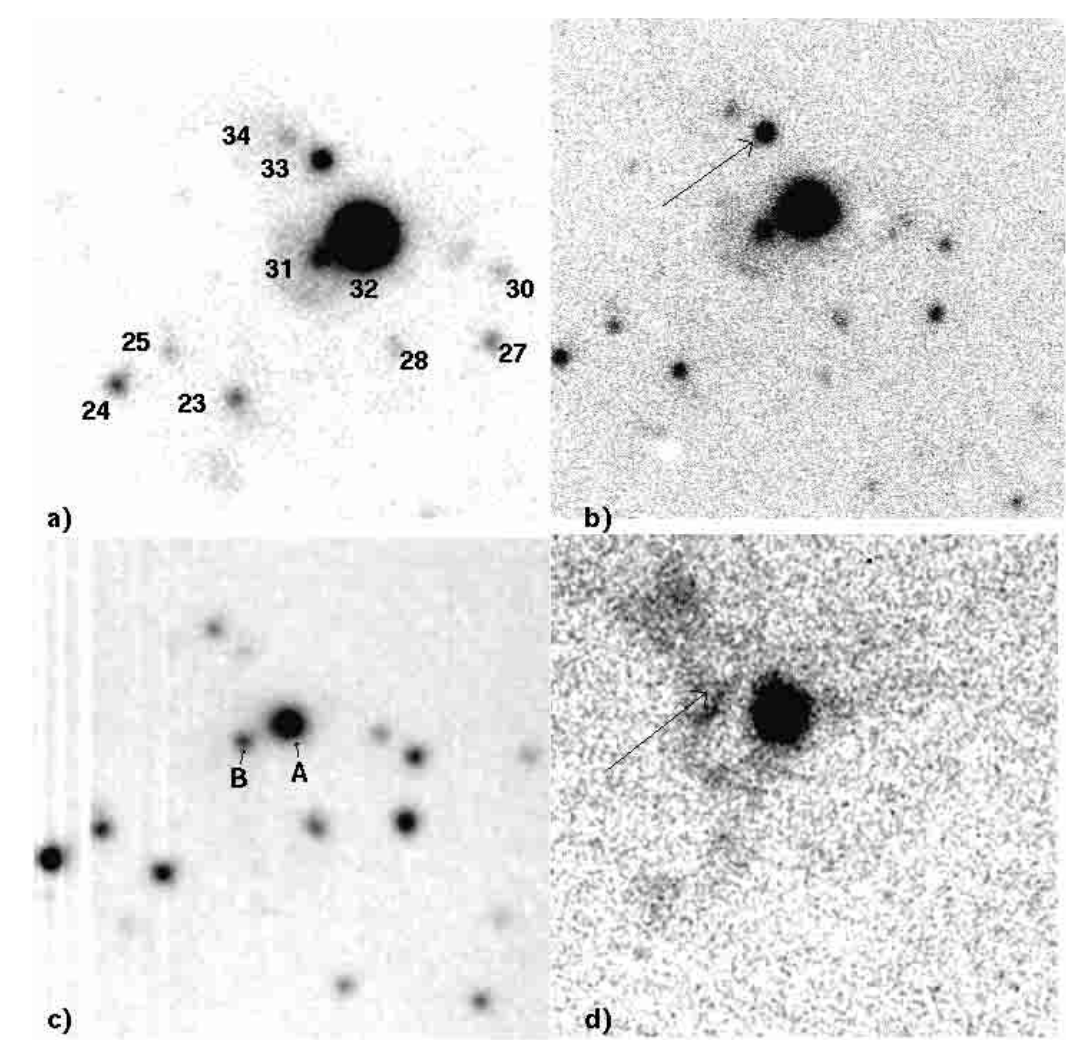

Fig. 1. Extracted images of monochromatic CCD frames in the optical and NIR continuum a) $K$-band image with stars labelled. b) $\lambda 2.13 \mu \mathrm{m}$, the red star is indicated by an arrow. c) $\lambda 5300 \AA$, the two components of $\mathrm{N} 88$ are labelled A and B. d) The $\mathrm{H}_{2}$ emitting region with its main $\mathrm{N}-\mathrm{S}$ filament and peak of intensity, is indicated by an arrow. The size of each field corresponds to $52^{\prime \prime} \times 51^{\prime \prime}$ or $\sim 15.8 \times 15.5 \mathrm{pc}$.

Table 2. Log of CCD images.

\begin{tabular}{|c|c|c|c|c|c|c|}
\hline Id. & Filter & & $\delta \lambda$ & $\underset{\text { (s) }}{\text { Expo. }}$ & Tel. & Date \\
\hline & $\begin{array}{l}\text { Cont }^{1} \\
\text { Cont }^{2} \\
\text { Cont } \\
\text { K }\end{array}$ & $\begin{array}{l}5300 \AA \\
2.13 \mu \\
2.19 \mu\end{array}$ & $\begin{array}{l}100 \AA \\
0.02 \mu \\
0.02 \mu\end{array}$ & $\begin{aligned} 2 & \times 600 \\
20 & \times 100 \\
20 & \times 100 \\
5 & \times 10\end{aligned}$ & $\begin{array}{l}\text { ESO - } \\
\text { VLT-IS }\end{array}$ & C 12 \\
\hline
\end{tabular}

${ }^{1}$ Roughly centered on the Johnson $V$ band, ${ }^{2}$ on N88A this filter mainly contains a HeI line, the $\mathrm{H}_{2} 1-0 \mathrm{~S}(1)$ line and continuum. In each image the seeing is $\sim 1^{\prime \prime} 7$.

\subsection{Photometry}

From the $K$ band and cont $_{5300}$ images the instrumental magnitudes of a few stars were computed using DAOPHOT/ALLSTAR (Stetson 1987) in a field of $50^{\prime \prime} \times 50^{\prime \prime}$ centered on N88A (Fig. 1a). The FWHM of N88A is $2.3^{\prime \prime}$ and the point spread function $(\mathrm{PSF})=1.75^{\prime \prime}$. Therefore the integrated magnitude of N88A was derived using circular apertures. Photometric calibration in the $K$ band was obtained using the reference star HD 7644 of $K$ magnitude $=9.27$ (van der Bliek et al. 1996), whereas for the cont $_{5300}$ we used the star of coordinates [1.24 04.89-7309 15.0 (J2000)] and $V=14.70$ found in Massey (2002). Table 3 shows magnitudes of stars in the region, taken from literature values, and the present cont $\lambda 5300$ and $K$ magnitudes (Cols. 15 and 16). Assigned spectral types are also shown. Table 3 also contains, as available, the $U B V$ photometry obtained by Massey (2002)
(Cols. 4-6) and the $I J H K$ photometry found in the infrared data base of Denis (Cioni et al. 2000) (Cols 7-9) and 2MASS (IPAC/UMass, 2000) (Cols. 10-12). In Cols. 13 and 14 are shown $V$ and Strömgren $y$ magnitudes from Wilcots (1994b) and HM99. The magnitudes derived by Wilcots and HM99 compared with Massey's photometry seem overestimated by at least one magnitude. Table 3 represents an extract of a larger VLT-ISAAC field for which the complete $K$ band photometry will be published in Paper II.

\section{Morphology}

\section{1. $\mathrm{H}_{2}$ emission associated with N88}

$\mathrm{H}_{2}$ emission has been detected in N88A. Detection was achieved by substracting the image in the $2.19 \mu \mathrm{m}$ filter, which allows the passage only of continuum radiation, from the image in the $2.13 \mu \mathrm{m}$ filter (see Sect. 2.2). Data were corrected for the different responses of the instrument through the filters, through observations of stars in the field. The resulting image is shown in Fig. 1d. The very precise removal of all stars in this image indicates accurate continuum subtraction. The strong emission directly over N88A has an unknown contribution from the HeI line at $2.11 \mu \mathrm{m}$. The $\mathrm{H}_{2}$ contribution will be discussed in Paper II. However around N88A there is highly extended emission, due purely to $\mathrm{H}_{2}$ in three roughly equally spaced arms. The length of each of these arms is 6 to $7 \mathrm{pc}$. The extent of the region containing $\mathrm{H}_{2}$ emission is similar to the extent of $\mathrm{H}_{2}$ emitting regions in highly luminous 
Table 3. N88A and a few neighbouring stars: values of the magnitudes $U, B, V, I, J, K, y$ (Strömgren $\lambda 5475 \AA$ ) found in the literature and values of the cont 5300 and $K$ magnitudes from the present work. Identification corresponds to the numbering in Fig. 1a.

\begin{tabular}{|c|c|c|c|c|c|c|c|c|c|c|c|c|c|c|}
\hline Id & $\alpha(2000) \delta$ & $\mid V^{\mathrm{M}}$ & $B-V U-B \mid I$ & & $J$ & $K$ & $\mid J^{2 \mathrm{M}}$ & $H$ & $K$ & $\mid V^{\mathrm{W}}$ & $\mid y^{\mathrm{H}}$ & $\lambda 5300^{\mathrm{P}}$ & $K$ & ype \\
\hline \begin{tabular}{ll|}
23 \\
24 \\
25 \\
27 \\
28 \\
30 \\
$31 \mathrm{~B}$ \\
32 \\
33 \\
34
\end{tabular} & $\mid \begin{array}{lllll}1 & 24 & 10.88-73 & 09 & 20 \\
1 & 24 & 13.94-73 & 09 & 19 \\
1 & 24 & 12.68-73 & 09 & 16 \\
1 & 24 & 04.89-73 & 09 & 15 \\
1 & 24 & 07.18-73 & 09 & 15 \\
1 & 24 & 04.79-73 & 09 & 07 \\
1 & 24 & 08.95-73 & 09 & 06 \\
1 & 24 & 07.88-73 & 09 & 04 \\
1 & 24 & 08.87-73 & 08 & 56 \\
1 & 24 & 09.88-73 & 08 & 53\end{array}$ & \begin{tabular}{l|l}
.0 & 14.70 \\
.6 & 14.61 \\
.3 & 14.67 \\
.5 & 12.18 \\
.1 &
\end{tabular} & 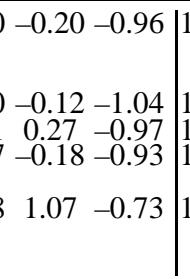 & $\begin{array}{l}15.36 \\
15.76 \\
15.60 \\
13.30\end{array}$ & $\begin{array}{l}15.67 \\
15.09 \\
15.76 \\
12.01\end{array}$ & $\begin{array}{l}7 \\
9 \\
5 \\
1 \\
10.97\end{array}$ & $\begin{array}{l}15.63 \\
16.39 \\
16.10 \\
15.12 \\
12.32\end{array}$ & $\begin{array}{l}15.94 \\
213.49 \\
12.01\end{array}$ & $\begin{array}{ll}1 & 15.61 \\
9 & 15.13 \\
4 & 15.57 \\
9 & 12.84 \\
1 & 11.19\end{array}$ & \begin{tabular}{l|l}
15.65 & $(\mathrm{BOIII})$ \\
14.91 & $(\mathrm{O} 9.5 \mathrm{III})$ \\
15.97 & $(\mathrm{BO})$ \\
1 & $16.88(\mathrm{BOIII})$ \\
7 & $16.01(\mathrm{BOV})$ \\
4 & \\
9 & $(\mathrm{O} 6)$ \\
& \\
16.54 & $(\mathrm{O} 9.5 \mathrm{I})$
\end{tabular} & $\begin{array}{l}15.28 \\
16.46 \\
15.68 \\
16.41 \\
\\
17.49 \\
18.02 \\
17.00\end{array}$ & 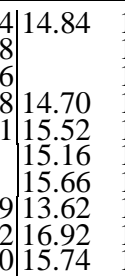 & $\begin{array}{l}15.54 \\
15.31 \\
16.20 \\
15.73 \\
16.44 \\
16.26 \\
15.37 \\
11.07 \\
14.54 \\
16.24\end{array}$ & $\begin{array}{ll}4 & -0.76 \\
1 & \\
5 & \\
3 & -1.03 \\
4 & -1.02 \\
5 & -1.10 \\
7 & 0.29 \text { O9.5 V } \\
7 & 2.55 \text { O6-O8V } \\
4 & 2.31 \text { G9-K1II } \\
4 & -0.50\end{array}$ \\
\hline
\end{tabular}

M, D and ${ }^{2 \mathrm{M}}$ correspond to Massey (2002), DENIS and 2MASS catalogues respectively.

${ }^{\mathrm{W}} V$ magnitudes and spectral types from Wilcots (1994b).

H, Strömgren $y$ (F547) magnitudes from HM99. For the stars 27, 28 and 32, spatially resolved into double or triple stars by the HST, the given magnitudes correspond to the sum of their components and are not affected by the brightness excess due to dust scattering.

p Present work.

massive star forming regions in the Galaxy, for example W51 (e.g. Hodapp \& Davis 2002), which has previously been likened to extremely active star-forming regions in other galaxies e.g. the 30 Doradus in the LMC (Hodapp \& Davis 2002). Further details of the $\mathrm{H}_{2}$ emitting region around N88A will be reported in Paper II, including $K$-band spectra of the brightest zones.

\subsection{Star 33}

Figure 1a shows, $\sim 8^{\prime \prime}$ to the north of N88A, a star labelled 33 which is brighter in the infrared (Figs. 1a,b) than in the visible (Fig. 1c). Using a distance modulus $m-M=19.09$ (Jacoby et al. 1990) and the tables of Schmidt-Kaler (1982) and Johnson \& Morgan (1953), we derive for star 33 an absolute magnitude $M_{V}$ of -2.17 and a $V-K$ of 2.31 (Table 3, Col. 17). From these parameters this star may be classified as a red supergiant (RSG) of type G9-K1 II.

\section{Physical conditions}

\subsection{Nebular reddening}

Using the $4^{\prime \prime} \times 4^{\prime \prime}$ slit aperture, the $\mathrm{H} \alpha / \mathrm{H} \beta$ ratio in N88A was found to be 4.2 , corresponding to a mean extinction $A_{V}$ of $1.14 \mathrm{mag}$. In N88B the $\mathrm{H} \alpha / \mathrm{H} \beta$ ratio is 3.39 ( $\left.A_{V} \sim 0.54 \mathrm{mag}\right)$. With a $2^{\prime \prime} \times 4^{\prime \prime}$ aperture the $\mathrm{H} \alpha / \mathrm{H} \beta$ ratio is $4.65\left(A_{V}=\right.$ $1.42 \mathrm{mag}$ ). Each ionizing source of $\mathrm{A}$ and $\mathrm{B}$ is of type $\mathrm{O}$ (Table 3). However the N88A/N88B ratio derived from cuts oriented N88A-N88B (Fig. 1c) is 45 (4.13 mag) in the cont $2.13 \mu$ or cont $_{2.19 \mu}$ images and 5.5 (1.85 mag) in the cont ${ }_{5300}$ image, as illustrated in Fig. 2. The magnitude excess of A in the cont $2.13 \mu$ image is estimated to be $\sim 2.3 \mathrm{mag}$, giving for N88A a mean $A_{V}$ of 3.44 mag. This strong mean $A_{V}$ found from NIR observations could be interpreted as dense internal dust associated with the central core of N88A and should have a local extinction $\gg 3.44$ mag. Our estimated extinction strengthens the argument for the strong $A_{V}$ of at least 6 mag estimated by HM99 at the position of the faintest star of the ionizing source.

In connection with nebular reddening, it is clear in the cont $_{15300}$ image (Fig. 1c) that the intensity ratio of the N88A

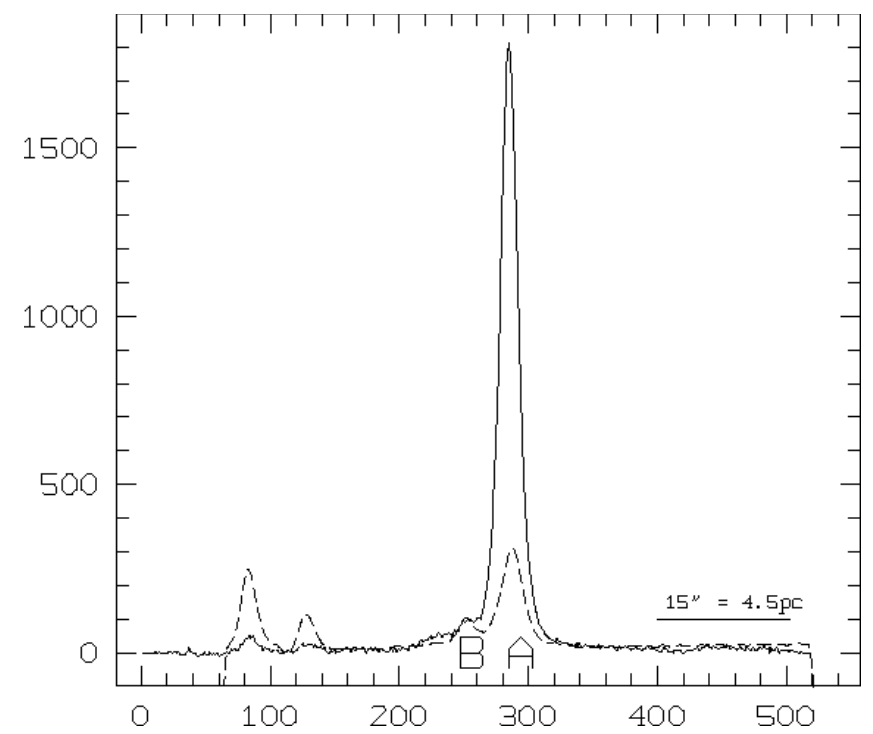

Fig. 2. Brightness distribution of the $5300 \AA$ (dashed line) and NIR continuum (solid line) images along the direction A-B.

to the N88B components is lower than in the cont $2.13 \mu$ image (Fig. 1b). Thus N88A is differentially more reddened than N88B, consistent with the greater contribution of longer wavelength emission in N88A.

\subsection{The ionizing source of $N 88 A$}

HST observations of HM99 have shown that the core A contains, in a diameter of $\sim 00^{\prime} 3$, two stars \#1 and \#2 and a third fainter star of $\sim 20 \mathrm{mag}$, the latter more dust embedded, just to the east of the brightest core A. These three stars of integrated magnitude $M_{5470}=17.5 \mathrm{mag}$ should be the sources responsible for the ionization of N88A. As our $V$ magnitudes of the stars compared with Wilcots (1994b) and HM99 appear one magnitude brighter, the integrated $V$ magnitude of the ionizing source should be $V \sim 16.5$ mag rather than $V \sim 17.5$ obtained by HM99 above the nebular continuum. Corrected for the reddening $\left(A_{V}=3.70 \mathrm{mag}\right.$, Sect. 4.1), the $V$ magnitude is expected to be 12.8 mag. Using Schmidt-Kaler's tables and a distance 


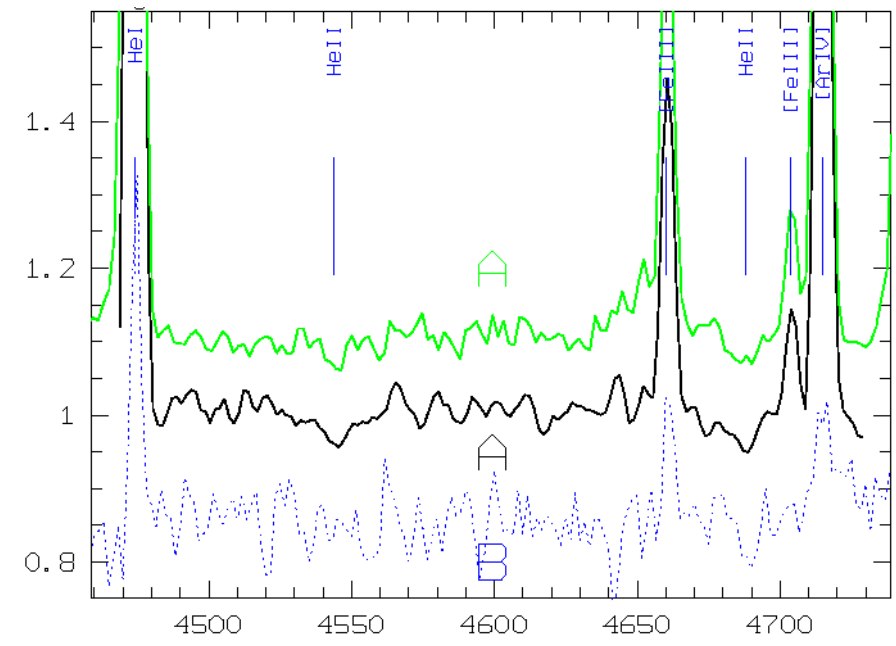

Fig. 3. E-W rectified composite spectra of the ionizing source embedded in the core N88A, in a 4450-4750 $\AA$ wavelength range obtained in 1991 (grey line) and 1988 (black line). The spectrum of the component B is also presented (dotted line). The continuum level is normalized to unity.

modulus of 19.09, such a magnitude suggests that the ionizing source could be a very early star of type O3.

\subsubsection{Spectral type derived from $N_{\text {Lyc }}$}

The Lyman continuum photon rate in photons per second, $N_{\text {Lyc }}$, produced by stars surrounded by an HII region can be derived from the flux of $\mathrm{H} \alpha$ photons $F_{\mathrm{H} \alpha}$ (Devereux \& Scowen 1994)

$N_{\text {Lyc }}=0.83 \times 10^{62} F_{\mathrm{H} \alpha} D^{2}$,

where $F_{\mathrm{H} \alpha}$ should be corrected for reddening and $D \sim 0.065$ is the distance in Mpc. The $N_{\text {Lyc }}$ value obtained can be expressed in terms of equivalent number of ionizing stars of a given spectral type. Using an $F_{\mathrm{H} \beta}$ of $4.14 \times 10^{-12} \mathrm{erg} \mathrm{s}^{-1} \mathrm{~cm}^{-2}$, uncorrected for reddening, obtained through an aperture of $4^{\prime \prime} \times 4^{\prime \prime}$ and a logarithmic reddening correction $c(\mathrm{H} \beta)$ of 0.52 (see Table 4), we derived a $N_{\text {Lyc }}$ of $1.372 \times 10^{49} v \mathrm{~s}^{-1}$. This indicates that the whole nebula should be excited by a source of equivalent type corresponding to an O7 V star (Vacca et al. 1996) or an O6.5 V (Panagia 1973). $N_{\text {Lyc }}$ should be a lower limit as a fraction of the ionizing photons should be absorbed by dust grains inside the nebula and another fraction might escape from the nebula as is the case in NGC 346 (Relano et al. 2002).

\subsubsection{Spectral type derived from spectroscopy}

To determine the spectral type of the faint embedded ionizing source, we used a spectrum resulting from an average of five east-west $1800 \mathrm{~s}$ exposures. A single 1D-spectrum was extracted by summing a pixel range along the $2^{\prime \prime}$ slit width, corresponding to $4^{\prime \prime}$. This $2^{\prime \prime} \times 4^{\prime \prime}$ aperture was centered on N88A, which is of $F W H M \sim 2.4^{\prime \prime}$ in $\mathrm{H} \beta$. The two components $\mathrm{A}$ and $\mathrm{B}$ are spatially resolved and the contribution of $\mathrm{B}$ is negligible. In Fig. 3 the E-W spectrum of the component $A$, in the range 4450-4750 $\AA$, clearly shows the HeII absorption lines $\lambda 4541$ and 24686 . It is not possible to discriminate between absorption and emission in the He I $\lambda 4471$ line, even if this line is analyzed with the higher spectral resolution spectrum $\delta \lambda=1.1 \AA$ obtained in 1995 (Table 1). Comparing the composite spectrum shown in Fig. 3 with that in the atlas of Walborn \& Fitzpatrick (1990), we estimate that the exciting source of N88A has an equivalent spectral type of O6-O8 V.

The spectral type classification is strengthened by the NIR spectrum (Paper II), where the ratio of $2.11 \mu \mathrm{m} \mathrm{He}$ I to Br $\gamma=$ 0.06. According to Hanson et al. (2002), this ratio is consistent with an ionizing source of spectral type $\leq 07$ V. Data in Fig. 3 also show the spectrum of N88B and in addition allow the classification of the ionizing star of $\mathrm{N} 88 \mathrm{~B}$, which appears to be a late $\mathrm{O}$ star.

\subsection{Temperature and density}

Physical parameters were computed using the 1996 version of Abelion developed by Stasinska. This program uses a fivelevel atom code and the atomic data are described by Stasinska $\&$ Leitherer (1996). The emission-line intensities (Fig.4) were corrected for reddening using the observed $\mathrm{H} \gamma / \mathrm{H} \beta$ ratios and Seaton's law (Seaton 1979) for N88A, whereas for the fainter component $\mathrm{N} 88 \mathrm{~B}$ we used the $\mathrm{H} \alpha / \mathrm{H} \beta$ ratio. The reddeninguncorrected lines normalized to $\mathrm{H} \beta$ are listed in Table 4 with the corresponding signal to noise ratio $(S / N)$ derived from the method given by Newberry (1994).

Four electron temperatures were derived, associated with different ionization regions in the nebula. These temperatures were computed from the following line intensity ratios: $T_{\mathrm{e}}\left[\mathrm{O}\right.$ III] $\lambda 4363 / \lambda \lambda(4959+5007), T_{\mathrm{e}}\left[\begin{array}{ll}\mathrm{N} & \text { II }]\end{array}\right.$ $\lambda 5755 / \lambda \lambda(6548+6584), T_{\mathrm{e}}[\mathrm{O}$ II $] \lambda 3727 / \lambda \lambda(7319+7330)$ and [S III] $\lambda 6312 / \lambda 9532$. Two electron densities were determined from the [S II] $\lambda 6717 / \lambda 6731$ and [Cl III] $\lambda 5517 / \lambda 5537$ ratios. In the determination of the physical parameters obtained from $\mathrm{S}$ and $\mathrm{N}$ lines, only the [O III] $\lambda 4363 / \lambda \lambda(4959+5007)$ and [S II] $\lambda 6717 / \lambda 6731$ ratios were used. The mean electronic densities, $N_{\mathrm{e}}$, and the mean electron temperatures $T_{\mathrm{e}}[\mathrm{O} \mathrm{III}]$, are shown in the last five rows of Table 4 as well as temperatures and densities obtained with other ratios, for comparison.

\subsection{Mass of ionized gas}

Density diagnostics such as [SII] line ratios measure the density of the matter $N_{\mathrm{e}}([\mathrm{S}$ II] $)$ while the mean electron density $<N_{\mathrm{e}}>$ (Pereira 2000), as derived from $N_{\mathrm{Lyc}}$, includes the medium empty of electrons.

$<N_{\mathrm{e}}>=\left(\frac{6 N_{\mathrm{Lyc}}}{\pi \alpha_{\mathrm{B}} D^{3}}\right)^{0.5}$,

where $\alpha_{\mathrm{B}}$ is the recombination coefficient for Case B (Osterbrock 1974) and $D=1.2 \mathrm{pc}$, the diameter of the HII region as measured using the $4^{\prime \prime}$ slit aperture. The ratio of the square of these two electron densities (Peimbert 1966) is taken to be the filling factor $\mathrm{f}$ and is given by the relation

$f=\frac{<N_{\mathrm{e}}^{2}>}{N_{\mathrm{e}}([\mathrm{SII}])^{2}}$ 


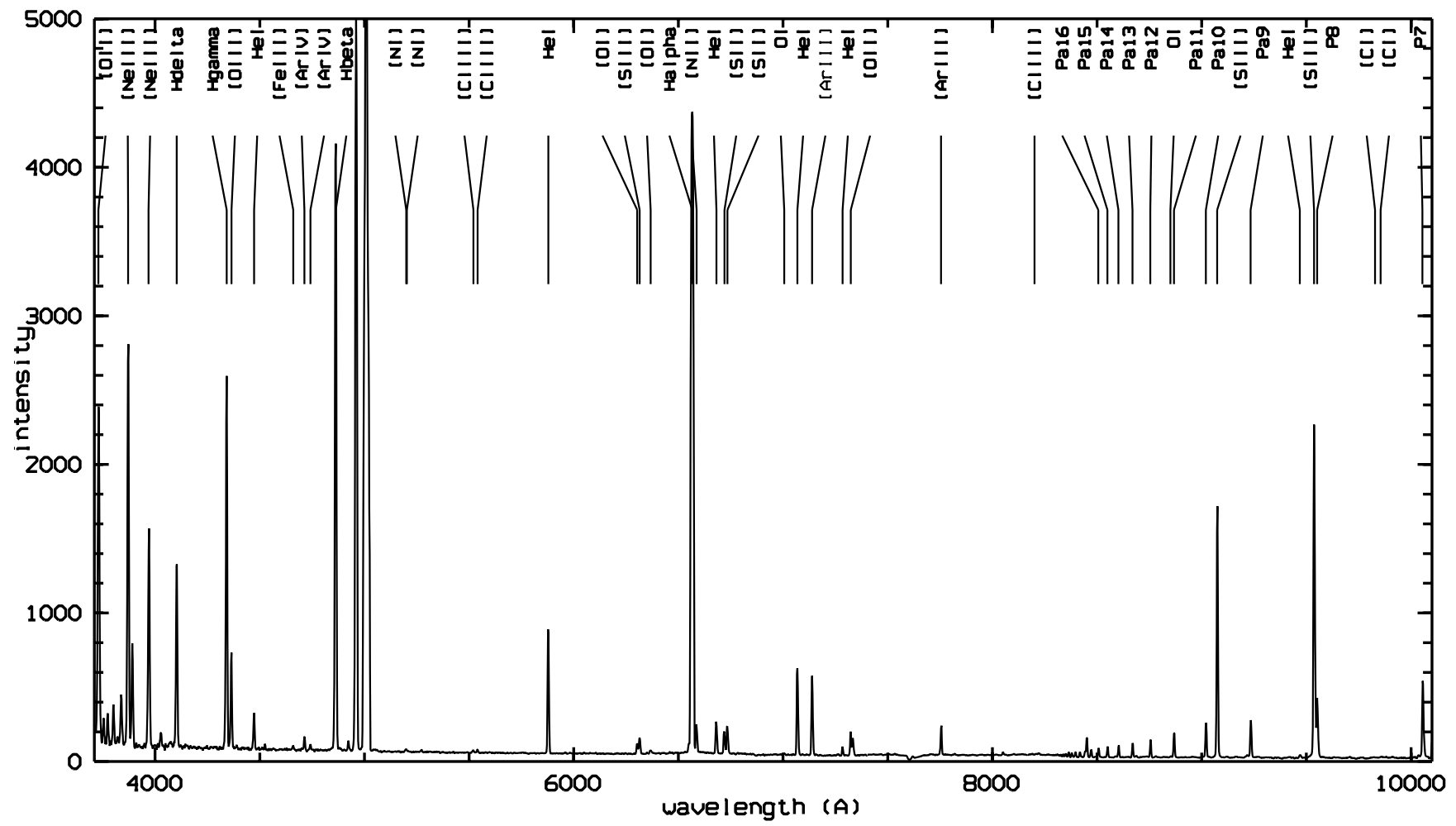

Fig. 4. E-W matched red and blue CCD spectra obtained in 1997 (long exposures) through an $4^{\prime \prime} \times 4^{\prime \prime}$ aperture. The intensity is in units of $10^{-16} \operatorname{ergs~cm}^{-2} \AA^{-1}$.

The mass of ionized gas $\left(M_{\mathrm{HII}}\right)$, in units of solar masses, is derived from $N_{\text {Lyc }}$ using the relation

$M_{\mathrm{HII}}=\frac{4 \pi r_{\mathrm{S}}^{3}<N_{\mathrm{e}}>m_{\mathrm{H}}}{3}$,

where $m_{\mathrm{H}}$ is the mass of the hydrogen atom and $r_{\mathrm{S}}$ the Strömgren radius. We find a filling factor $f=0.5$ and a mass of ionized gaz of $\sim 32 M_{\odot}$. This mass is smaller than that found by HM99 $\left(45 M_{\odot}\right)$ but lies in the estimated range of $20 M_{\odot}$ to $50 M_{\odot}$ of Testor \& Pakull (1985). Any discrepancy may be attributed to the inhomogeneous dust content of N88A, which makes the reddening determination difficult.

\section{Abundances}

$\mathrm{N} 88 \mathrm{~A}$ is an HII region well adapted to computation of abundances. It is bright and its angular size is such that the Strömgren sphere fills the slit of the spectrograph.

\subsection{Ionic and elemental abundances}

Table 5 lists the ionic abundances of $\mathrm{O}^{+}, \mathrm{O}^{++}, \mathrm{S}^{+}$and $\mathrm{S}^{++}$for component A. The total abundances of Ne, N, S and Ar have been calculated using ionization corrector factors (ICFs) described by Vermej \& van der Hulst (2002, Eqs. (6)-(8)) with $\alpha=2.5$, using the derived $T_{\mathrm{e}}[\mathrm{O} \mathrm{III}]$ and $N_{\mathrm{e}}[\mathrm{S}$ II $]$ values for the nebula. Results are presented in Table 6. For comparison, Table 6 also contains the latest average HII abundances in the SMC derived by Reyes (1999) and Garnett (1999) as well as the N88A abundances derived from the studies of Kurt et al. (1999) and Vermej (2002). Small discrepancies are noticed between the abundances derived from 1991 and 1997 observations as well as between the mean abundances given by Reyes (1999) and Garnett (1999). Despite our small error bars, it is difficult to maintain that the scatter of results is physically real. Except for $\mathrm{S}$ that appears underabundant, all our variations are within the errors given by most of the different authors. Our detection of a weak emission feature at [CI] $\lambda 9850$ (Table 4) should, according to Rudy et al. (2001), strengthen the conclusion that N88A is carbon rich in nature, as established by Kurt et al. (1999).

Vilchez \& Pagel (1988) defined a radiation softness parameter from the ionic ratio

$\eta=\frac{\mathrm{O}^{+} / \mathrm{O}^{++}}{\mathrm{S}^{+} / \mathrm{S}^{++}}$

Using their Fig. 1, and Fig. 6 of Garnett (1989) we find that the $\eta$ parameter for $\mathrm{N} 88 \mathrm{~A}$ is consistent with an effective temperature of $\sim 50000 \mathrm{~K}$ corresponding to an ionizing source of spectral type O3-O4 V (Vacca et al. 1996) in agreement with the suggestion in Sect. 4.2. This very early type implies that an additional star of type equal or hotter than an $\mathrm{O} 4 \mathrm{~V}$ is likely to be hidden in the faintest dusty component. The spectral type $\mathrm{O} 7 \mathrm{~V}$ derived from our composite spectrum should correspond to the other two bright stars \#1 and \#2 composing the ionizing source. 
Table 4. Forbidden and permitted SMC N88A \& B emission line intensities normalized to $\mathrm{H} \beta$.

\begin{tabular}{|c|c|c|c|c|c|c|c|c|c|c|c|c|c|}
\hline$\lambda$ & Ion & $\begin{array}{l}I \lambda / \mathrm{H} \beta \\
\mathrm{N} 88 \mathrm{~A}^{91}\end{array}$ & $S / N$ & $\begin{array}{l}I \lambda / \mathrm{H} \beta \\
\mathrm{N}^{2} 8 \mathrm{~B}^{91}\end{array}$ & $\begin{array}{l}I \lambda / \mathrm{H} \beta \\
\mathrm{N} 88 \mathrm{~A}^{97}\end{array}$ & $S / N$ & $\lambda$ & Ion & $\begin{array}{l}I \lambda / \mathrm{H} \beta \\
\mathrm{N} 88 \mathrm{~A}^{91}\end{array}$ & $S / N$ & $\begin{array}{l}I \lambda / \mathrm{H} \beta \\
\mathrm{N} 88 \mathrm{~B}^{91}\end{array}$ & $\begin{array}{l}I \lambda / \mathrm{H} \beta \\
\mathrm{N} 88 \mathrm{~A}^{97}\end{array}$ & $S / N$ \\
\hline 3727 & [O II] & 0.2412 & 457 & 1.9000 & 0.5304 & 3103 & 5958 & Si II & 0.0013 & 19 & & & \\
\hline 3868 & [Ne III] & 0.3955 & 670 & 0.2099 & 0.5495 & 417 & 5979 & Si II & 0.0012 & 17 & & & \\
\hline 4072 & [S II] & 0.0071 & 37 & 0.0075 & 0.0046 & 3 & 6046 & O I & 0.0011 & 17 & & & \\
\hline 4076 & [S II] & 0.0024 & 8 & & & & 6300 & [O I] & 0.0131 & 130 & 0.0387 & 0.0136 & 45 \\
\hline 4100 & $\mathrm{H} \delta$ & 0.1893 & 500 & 0.2055 & 0.2140 & 220 & 6311 & [S III] & 0.0238 & 197 & 0.0164 & 0.0220 & 82 \\
\hline 4157 & O II & 0.0003 & 2 & & & & 6347 & Si II & 0.0013 & 14 & & & \\
\hline 4267 & C II & 0.0002 & 1 & & & & 6364 & {$[\mathrm{O} \mathrm{I}]$} & 0.0043 & 54 & & & \\
\hline 4287 & {$[\mathrm{Fe} \mathrm{II}]$} & 0.0010 & 7 & & & & 6563 & $\mathrm{H} \alpha$ & & & 3.3900 & 4.15 & \\
\hline 4340 & $\mathrm{H} \gamma$ & 0.3907 & 780 & 0.4115 & 0.4400 & 458 & 6584 & [N II] & 0.0306 & 197 & 0.1204 & 0.0461 & 91 \\
\hline 4363 & [O III] & 0.1095 & 338 & 0.0526 & 0.1020 & 130 & 6678 & $\mathrm{He} \mathrm{I}$ & 0.0433 & 367 & 0.0290 & 0.0438 & 113 \\
\hline 4415 & O II & 0.0012 & 7 & & & & 6717 & [S II] & 0.0214 & 230 & 0.1273 & 0.0301 & 94 \\
\hline 4471 & $\mathrm{He} \mathrm{I}$ & 0.0394 & 170 & 0.0332 & 0.0519 & 48 & 6731 & [S II] & 0.0325 & 288 & 0.1124 & 0.0393 & 109 \\
\hline 4649 & O II & 0.0003 & 3 & & & & 7002 & O I & 0.0017 & 20 & & & \\
\hline 4658 & {$[\mathrm{Fe} \mathrm{III}]$} & 0.0055 & 33 & & & & 7135 & [Ar III] & 0.1297 & 729 & 0.0891 & 0.1022 & 259 \\
\hline 4701 & {$[\mathrm{Fe} \mathrm{III]}]$} & 0.0016 & 14 & & & & 7256 & $\mathrm{O} I$ & 0.0018 & 20 & & & \\
\hline 4711 & {$[\mathrm{Ar} \mathrm{IV}]^{b}$} & 0.0090 & 90 & 0.0043 & 0.0171 & 28 & 7325 & [O II] & 0.0525 & 268 & 0.0861 & 0.0495 & 121 \\
\hline 4740 & [Ar IV] & 0.0068 & 58 & 0.0035 & 0.0068 & & 8413 & p19 & & & & 0.0059 & 8 \\
\hline 4754 & [Fe III] & 0.0015 & 6 & & & & 8467 & p17 & & & & 0.0090 & 13 \\
\hline 4769 & {$[\mathrm{Fe} \mathrm{III}]$} & 0.0004 & 3 & & & & 8502 & p16 & & & & 0.0101 & 15 \\
\hline 4861 & $\mathrm{H} \beta$ & 1. & 1690 & & 1. & 964 & 8545 & p15 & & & & 0.0118 & 18 \\
\hline 4881 & {$[\mathrm{Fe} \mathrm{III}]$} & 0.0020 & 20 & & & & 8598 & p14 & & & & 0.0128 & 20 \\
\hline 4959 & [O III] & 2.5446 & 2700 & & & & 8665 & p13 & & & & 0.0161 & 25 \\
\hline 5007 & [O III] & 7.62 & & 4.08 & 7.44 & & 8750 & p12 & & & & 0.0182 & 30 \\
\hline 5056 & Si II & 0.0013 & 16 & & & & 8863 & p11 & & & & 0.0284 & 40 \\
\hline 5200 & {$[\mathrm{~N} \mathrm{I}]$} & 0.0029 & 28 & & & & 9015 & p10 & & & & 0.0408 & 50 \\
\hline 5270 & {$[\mathrm{Fe}$ III $]$} & 0.0027 & 32 & & & & 9069 & [S III] & & & & 0.3007 & 300 \\
\hline 5299 & O I & 0.0003 & 3 & & & & 9229 & p9 & & & & 0.0455 & 57 \\
\hline 5518 & [Cl III] & 0.0039 & 41 & & & & 9473 & $\mathrm{He} \mathrm{I}$ & & & & 0.0065 & 7 \\
\hline 5538 & [Cl III] & 0.0041 & 47 & & & & 9532 & {$[\mathrm{~S} \text { III }]^{a b s}$} & & & & 0.7456 & 400 \\
\hline 5755 & {$[\mathrm{~N} \mathrm{II}]$} & 0.0009 & 5 & 0.0040 & 0.0009 & & 9850 & {$[\mathrm{C} \mathrm{I}]$} & & & & 0.0015 & 2 \\
\hline 5876 & He I & 0.1793 & 775 & 0.1384 & 0.1613 & 406 & 10057 & p7 & & & & 0.1083 & 125 \\
\hline$F(\mathrm{H} \beta)$ & & & & & $4.06^{u}$ & & & & & & & & \\
\hline$c(\mathrm{H} \beta)$ & & 0.65 & & 0.29 & 0.52 & & & & & & & & \\
\hline$N_{\mathrm{e}}[\mathrm{S} \mathrm{II}]$ & $\left(\mathrm{cm}^{-3}\right)$ & 2870 & & 369 & 1710 & & & & & & & & \\
\hline$N_{\mathrm{e}}[\mathrm{Cl} \mathrm{III}]$ & $\left(\mathrm{cm}^{-3}\right)$ & 3623 & & & & & & & & & & & \\
\hline$N_{\mathrm{e}}[\mathrm{Ar}$ IV] & $\left(\mathrm{cm}^{-3}\right)$ & 5840 & & & & & & & & & & & \\
\hline$T_{\mathrm{e}}(\mathrm{K})$ & [O III] & 14100 & & 13100 & 14300 & & & & & & & & \\
\hline$T_{\mathrm{e}}(\mathrm{K})$ & {$[\mathrm{N} \mathrm{III}]$} & & & & & & & & 12900 & & 13200 & 12200 & \\
\hline
\end{tabular}

Note. The E-W long slit centered on A crosses B in its northern part.

${ }^{b}$ As the line is affected by blending, an additional spectrum was obtained with a $F W H M=1.1 \AA$ through the same slit width giving an [Ar IV] $\lambda 4711 /[\mathrm{Ar}$ IV] $\lambda 4711+\mathrm{HeI} \lambda 4713$ ratio of 0.47 .

abs Since [SIII] $\lambda 9532$ is affected by telluric absorption its intensity has been estimated from the intensity of $\lambda 9069$ and taking into account that the A value of $\lambda 9532=0.0576$ and that the A value of $\lambda 9069=0.0221$ (Mendoza 1983).

${ }^{u}$ In units of $10^{-12} \mathrm{ergs} \mathrm{s}^{-1} \mathrm{~cm}^{-2}$, the equivalent width of $\mathrm{H}(\beta)$ is $415 \AA$.

91, 971991 and 1997 observations.

\section{Summary of results}

Based on optical spectra and NIR data:

- An $\mathrm{H}_{2}$ emitting region was detected in the direction of N88A, composed of 3 main filaments, with the strongest oriented N-S and located a few arcsecs to the east of N88A.

- The integrated magnitude of the ionizing source of N88A was found to be one $V$ mag brighter than the value previously estimated.
Table 5. N88A Ionic abundances: $10^{-8} \mathrm{X} / \mathrm{H}$.

\begin{tabular}{lll}
\hline \hline Abundance ratio & $\mathrm{N}_{88 \mathrm{~A}^{91}}$ & $\mathrm{~N} 88 \mathrm{~A}^{97}$ \\
\hline $\mathrm{O}^{+} / \mathrm{H}$ & 534 & 880 \\
$\mathrm{O}^{++} / \mathrm{H}$ & 8820 & 8320 \\
$\mathrm{~S}^{+} / \mathrm{H}$ & 5.10 & 6.07 \\
$\mathrm{~S}^{++} / \mathrm{H}$ & 104 & 103 \\
\hline
\end{tabular}


Table 6. Element abundances in N88 A \& B in the form $(12+\log [N(\mathrm{X}) / N(\mathrm{H})])$, derived from spectra obtained with $2^{\prime \prime}$ and 4 " slit widths.

\begin{tabular}{|c|c|c|c|c|c|c|c|c|}
\hline Name & $\mathrm{O}$ & $\mathrm{N}$ & $\mathrm{Ne}$ & $\mathrm{Ar}$ & $\mathrm{S}$ & $\mathrm{He}$ & $c(\mathrm{H} \beta)$ & $\begin{array}{l}\text { slit } \\
\left({ }^{\prime \prime}\right)\end{array}$ \\
\hline $\begin{array}{l}\mathrm{N} 88 \mathrm{~A}_{\mathrm{av}}^{91} \\
\mathrm{~N} 88 \mathrm{~B}_{\mathrm{ay}}^{91} \\
\mathrm{~N} 88 \mathrm{~A}^{9 y}\end{array}$ & $\begin{array}{l}7.98 \pm 0.02 \\
7.97 \pm 0.04 \\
7.98 \pm 0.04\end{array}$ & $\begin{array}{l}6.56 \pm 0.05 \\
6.49 \pm 0.06 \\
6.46 \pm 0.06\end{array}$ & $\begin{array}{l}7.23 \pm 0.04 \\
7.14 \pm 0.05 \\
7.35 \pm 0.04\end{array}$ & $\begin{array}{l}5.68 \pm 0.04 \\
5.72 \pm 0.06 \\
5.62 \pm 0.05\end{array}$ & $\begin{array}{l}6.36 \pm 0.05 \\
6.35 \pm 0.06\end{array}$ & $\begin{array}{l}10.90 \\
10.93 \\
10.90\end{array}$ & $\begin{array}{l}0.65 \\
0.29 \\
0.52\end{array}$ & $\begin{array}{l}2 \times 4 \\
2 \times 4 \\
4 \times 4\end{array}$ \\
\hline $\begin{array}{l}\mathrm{SMC}^{\mathrm{RU}} \\
\mathrm{SMC}^{\mathrm{D}} \\
\mathrm{SMC}^{\mathrm{RE}} \\
\mathrm{SMC}^{\mathrm{G}}\end{array}$ & $\begin{array}{l}8.03 \pm 0.10 \\
8.02 \pm 0.08 \\
7.96 \\
8.0\end{array}$ & $\begin{array}{l}6.63 \pm 0.20 \\
6.46 \pm 0.12 \\
6.55 \\
6.5\end{array}$ & $\begin{array}{l}7.27 \pm 0.20 \\
7.22 \pm 0.12 \\
7.17 \\
7.2\end{array}$ & $\begin{array}{l}5.81 \pm 0.08 \\
5.78 \pm 0.12 \\
5.72 \\
5.9\end{array}$ & $\begin{array}{l}6.32 \\
6.3\end{array}$ & $\begin{array}{l}10.91 \\
10.92 \\
10.91\end{array}$ & & \\
\hline $\begin{array}{l}\text { N88A } A^{\mathrm{K}} \\
\mathrm{N} 88 \mathrm{~A}^{\mathrm{VH}}\end{array}$ & $\begin{array}{l}8.05 \pm 0.08 \\
7.89\end{array}$ & $\begin{array}{l}6.59 \pm 0.08 \\
6.38\end{array}$ & $\begin{array}{l}7.26 \pm 0.05 \\
6.97\end{array}$ & $\begin{array}{l}5.69 \pm 0.07 \\
5.66\end{array}$ & $\begin{array}{l}6.42 \pm 0.11 \\
6.08\end{array}$ & 10.91 & 0.66 & $\begin{array}{c}2 \times 330 \\
1.7\end{array}$ \\
\hline
\end{tabular}

D Dufour et al. (1982), ${ }^{\mathrm{G}}$ Garnett (1999), ${ }^{\mathrm{K}}$ Kurt et al. (1999), ${ }^{\mathrm{Ru}}$ Russell et al. (1990), ${ }^{\mathrm{R}}$ Reyes (1999), ${ }^{\mathrm{VH}}$ Vermeij (2002), ${ }_{\text {av }}$ averaged frames from 1991 observations.

${ }^{97} 1997$ observations.

- The first composite spectrum of N88A is presented, showing the HeII absorption lines. This spectrum allows classification of the complex ionizing source as 06-O8 V. This result is in agreement with the exciting source stellar type derived from the $\mathrm{H} \beta$ luminosity and the $\mathrm{HeI} / \mathrm{Br} \gamma$ ratio but does not allow us to determine with certainty if the source contains a very early O star, as suspected from the strong extinction detected from NIR observations and the $\eta$ parameter.

- The presence of the red super-giant (\#33) is consistent with the OB star population towards N88.

- We calculate, using accurate electron temperatures, electron densities and empirical ICFs, new elemental abundances of $\mathrm{He}, \mathrm{O}, \mathrm{Ne}, \mathrm{S}$ and Ar. These values are found to be consistent with the mean average of the SMC. However our values present small discrepancies both with average SMC abundances and with earlier estimates of N88A abundances, which themselves display differences. The likely cause of discrepancies lies in uncertainties in reddening corrections.

- We confirm the carbon rich nature of N88A (Kurt et al. 1999), through the detection of the [CI] $\lambda 9850$ emission line. In conclusion we note that the nature of the exciting sources of N88A and B could be more precisely determined using high spatial resolution, NIR imaging and spectroscopy, for example with the adaptive optics capability which has recently become available on UT4 (NAOS-CONICA). In Paper II, we extend the present study to consider in detail the nature of the $\mathrm{H}_{2}$ emitting region in N88.

Acknowledgements. We thank the referee, Manuel Peimbert, for a careful reading of the manuscript and several suggestions, which significantly improved the paper. D.F would like to acknowledge the support of the Aarhus Centre for Atomic Physics (ACAP), funded by the Danish Basic Science Foundation. We should also like to thank the Director and Staff of ESO especially for making possible the IR observations reported in this paper.

\section{References}

Churchwell, E. 1990, A\&ARv, 2, 79

Cioni, M.-R., Loup, C., Habing, H. J., et al. 2000, A\&AS, 144, 235

Devereux, N. A., \& Scowen, P. A. 1994, AJ, 108, 1244

Dufour, R. J., Shields, G. A., \& Talbot, R. J. 1982, ApJ, 339, 872
Garnett, D. 1989, ApJ, 345, 282

Garnett, D. 1999, in IAU Symp., 190, 266

Hanson, M. M., Luhman, K. L., \& Rieke, G. H. 2002, ApJS, 138, 35

Henize, K. G. 1956, ApJS, 2, 315

Heydari-Malayeri, M., \& Testor, G. 1982, A\&A, 111, L11

Heydari-Malayeri, Charmandaris, V., Deharveng, et al. 2002, in IAU Symp., 212, 553

Heydari-Malayeri, Charmandaris, V., Deharveng, L., Rosa, M. R., \& Zinneker, H. 1999, A\&A, 347, 841

Hodapp, K. W., \& Davis, C. J. 2002, ApJ, 575, 291

Israel, F. P., \& Koorneef, J. 1988, A\&A, 190, 21

Israel, F. P., \& Koorneef, J. 1991, A\&A, 248, 404

IPAC/Umass, http://irsa.ipac.caltech.edu/applications/ 2MASS

Jacoby, G. H., Ciardullo, R., \& Walker, A. R. 1990, ApJ, 365, 471

Johnson, H. L., \& Morgan, W. W. 1953, ApJ, 117, 313

Kurt, C., Dufour, R., Garnett, D., et al. 1999, ApJ, 518, 246, 261

Massey, P. 2002, ApJS, 141, 81

Mendoza, C. 1983, in IAU Symp., 103, 143

Newberry, M. V. 1994, CCDA, 1, 34

Osterbrock, DE. 1974, Astrophysics of Gaseous Nebulae (Freeman: San Francisco)

Oke, J. B. 1974, ApJS, 27, 21

Panagia, N. 1973, AJ, 78, 929

Peimbert, M. 1966, ApJ, 145, 75

Pereira, C. B. 2000, AJ, 119, 63

Relano, M., Peimbert, M., \& Beckman, J. 2002, ApJ, 564, 704

Reyes, C. 1999, in IAU Symp., 190, 282

Rudy, R., Lynch, David., Mazuk, S., Puetter, R. C., \& Dearborn, D. S. P. 2001, AJ, 121, 362

Russell, S. D., \& Dopita, M. A. 1990, ApJS, 74, 93

Schmidt-Kaler, T. 1982, Landoldt-Bornstein, New Series, Group VI, 14

Stetson, P. B. 1987, PASP, 99, 191

Seaton, M. J. 1979, MNRAS, 187, 73P

Stasinska, G., \& Leitherer, C. 1996, ApJS, 107, 661

Testor, G., \& Pakull, M. 1985, A\&A, 145, 170

Testor, G. 2001, A\&A, 372, 667

Testor, G., 2002, in IAU Symp., 212, 753

Vacca, W. D., Garmany, C. D., \& Shull, J. M. 1996, ApJ, 460, 914

van der Bliek, N. S., Manfroid, J., \& Bouchet, P. 1996 A\&AS, 119, 547

Vermeij, R., \& van der Hulst, J. M. 2002, A\&A, 391, 1081

Vilchez, J. M., \& Pagel, B. E. J. 1988, MNRAS, 231, 257

Walborn, N., \& Fitzpatrick, E. 1990, PASP, 102, 379

Walborn, N., \& Parker, J. 1992, ApJ, 399, 87

Wilcots, E. M. 1994a, AJ, 107, 1338

Wilcots, E. M. 1994b, AJ, 108, 1674 